\title{
Studies on the Existence of Unstable Oscillatory Patterns Bifurcating from Hopf Bifurcations in a Turing Model
}

\author{
Yan Zhang ${ }^{1,2}$ and Zhenhua Bao ${ }^{2}$ \\ ${ }^{1}$ School of Control Science and Engineering, Dalian University of Technology, Dalian 116024, China \\ ${ }^{2}$ School of Mathematics, Liaoning Normal University, Dalian 116029, China \\ Correspondence should be addressed to Zhenhua Bao; zhhbao@126.com
}

Received 15 November 2013; Revised 28 April 2014; Accepted 9 May 2014; Published 20 May 2014

Academic Editor: Laura Rebollo-Neira

Copyright (c) 2014 Y. Zhang and Z. Bao. This is an open access article distributed under the Creative Commons Attribution License, which permits unrestricted use, distribution, and reproduction in any medium, provided the original work is properly cited.

\begin{abstract}
We revisit a homogeneous reaction-diffusion Turing model subject to the Neumann boundary conditions in the one-dimensional spatial domain. With the help of the Hopf bifurcation theory applicable to the reaction-diffusion equations, we are capable of proving the existence of Hopf bifurcations, which suggests the existence of spatially homogeneous and nonhomogeneous periodic solutions of this particular system. In particular, we also prove that the spatial homogeneous periodic solutions bifurcating from the smallest Hopf bifurcation point of the system are always unstable. This together with the instability results of the spatially nonhomogeneous periodic solutions by Yi et al., 2009, indicates that, in this model, all the oscillatory patterns from Hopf bifurcations are unstable.
\end{abstract}

\section{Introduction}

In 1952, Turing published his pioneering paper describing the chemical process between signaling molecules that spread away from their source to form a concentration gradient within a series of cells [1]. In this paper, Turing suggested that diffusion could destabilize an otherwise stable equilibrium of the reaction-diffusion system and lead to nonuniform spatial patterns [2-10].

The following model (which we called Turing model here) is one of the famous models used by Turing to argue the above-mentioned mechanism:

$$
\begin{gathered}
u_{t}=d_{u} \Delta u+s(u v-u-a), \quad x \in \Omega, t>0, \\
v_{t}=d_{v} \Delta v+s(b-u v), \quad x \in \Omega, t>0, \\
u(x, 0)=u_{0}(x), \quad v(x, 0)=v_{0}(x), \quad x \in \Omega, \\
\partial_{\nu} u=\partial_{\nu} v=0, \quad x \in \partial \Omega, t>0,
\end{gathered}
$$

where $\Omega$ is a bounded connected domain (the reactor) in $\mathbf{R}^{n}$ $(n \geq 1)$, with smooth boundary $\partial \Omega$; the reactor is assumed to be closed; thus reflexive Neumann boundary condition is imposed (here $\partial_{\nu} u$ is the outer normal derivative of $u$ ); here $u(x, t)$ and $v(x, t)$ are the morphogen concentration at the time $t$ and the positions $x, a$, and $b$ can be thought of as being a decay and growth rate of $u(x, t)$ and $v(x, t)$, respectively, and $s$ is the reaction rate.

Since the time when the model was proposed by Turing, many researchers have studied the model extensively (see [11] and the references therein for great details). However, they mostly focus on the pattern formations of Turing type.

It is well known that, apart from occurring Turing patterns, a reaction-diffusion equation system might also exhibit other types of patterns, say, oscillatory patterns.

In [12], the authors derived a simplified Hopf bifurcation theorem for the general semilinear reaction-diffusion equations on the one-dimensional domain and used the abstract theorem to prove the existence of oscillatory patterns emerging from Hopf bifurcations. Since then, more papers can be found in the existing literatures to study the existence of oscillatory patterns of the R-D systems by using Hopf bifurcation techniques, and we refer to [12-14] and the references therein for details.

To the best of our knowledge, in the existing literature, no papers were found to deal with the oscillatory pattern formation of this Turing model from purely analytical point of view. Thus, motivated by [12], we are interested in considering the existence of Hopf bifurcations and the corresponding 
periodic solutions by using the abstract bifurcation results in [12].

In order to set our problem in the framework of [12], we assume that the domain is one- dimensional spatial interval $(0, \xi \pi)$ with $\xi>0$. In this case, the system (1) is reduced to

$$
\begin{aligned}
u_{t} & =d_{u} u_{x x}+s(u v-u-a), \quad x \in(0, \xi \pi), t>0, \\
v_{t} & =d_{v} v_{x x}+s(b-u v), \quad x \in(0, \xi \pi), t>0, \\
u(x, 0) & =u_{0}(x), \quad v(x, 0)=v_{0}(x), \quad x \in(0, \xi \pi), \\
u^{\prime}(0, t) & =u^{\prime}(\xi \pi, t)=v^{\prime}(0, t)=v^{\prime}(\xi \pi, t)=0, \quad t>0 .
\end{aligned}
$$

We are going to show that if we choose $a$ as the bifurcation parameter, then the system might exhibit a sequence of Hopf bifurcation points, at which oscillatory patterns exist. By using the center manifold theory, normal form methods, and the abstract results in [12], we are able to prove that the spatially homogeneous periodic solutions bifurcating from the smallest Hopf bifurcation point are unstable. From [12], all the spatially nonhomogeneous periodic solutions are all unstable. Thus, our bifurcation results suggest that all the Hopf bifurcating periodic solutions are all unstable.

The rest of this paper is structured in the following way. In Section 2, we use the standard Hopf bifurcation theorem to prove the existence of the Hopf bifurcations of the system. In Section 3, we prove the instability of the spatially homogeneous periodic solutions bifurcating from the smallest Hopf bifurcation point. In Section 4, we draw our conclusions.

\section{Existence of Hopf Bifurcations}

In this section, we consider the following PDEs subject to Neumann boundary condition on spatial domain $\Omega=$ $(0, \xi \pi)$, with $\xi \in \mathbb{R}^{+}:$

$$
\begin{gathered}
\frac{\partial u}{\partial t}=d_{u} u_{x x}+s(u v-u-a), \quad x \in(0, \xi \pi), t>0, \\
\frac{\partial v}{\partial t}=d_{v} v_{x x}+s(b-u v), \quad x \in(0, \xi \pi), t>0, \\
u^{\prime}(0)=u^{\prime}(\xi \pi)=v^{\prime}(0)=v^{\prime}(\xi \pi)=0, \quad t>0 .
\end{gathered}
$$
with

System (3) has a unique equilibrium solution $\left(u_{a}, v_{a}\right)$,

$$
u_{a}=b-a, \quad v_{a}=\frac{b}{b-a},
$$

which is in the first quadrat if and only if $0<a<b$. In the following, we always assume that $0<a<b$ holds and choose $a$ as the bifurcation parameter.

Define the real Sobolev space in the following way:

$$
\begin{aligned}
X:=\{ & (u, v)^{T} \in H^{2}(0, \xi \pi) \\
& \left.\times H^{2}(0, \xi \pi):\left.\left(u_{x}, v_{x}\right)\right|_{x=0, \xi \pi}=0\right\},
\end{aligned}
$$

and $X_{\mathbb{C}}:=\left\{U=U_{1}+i U_{2}: U_{1}, U_{2} \in X\right\}$.
The aim of the whole paper is just to study the existence and the stability of the spatial homogeneous and nonhomogeneous periodic solutions of system (3) in the real-valued Sobolev space $X$ by using the Hopf bifurcation techniques.

To that end, we calculate the linearized operator of system (3) evaluated at $\left(u_{a}, v_{a}\right)$ which is given by

$$
L(a):=\left[\begin{array}{cc}
d_{u} \Delta+\frac{s a}{b-a}, & s(b-a) \\
-\frac{s b}{b-a}, & d_{v} \Delta-s(b-a),
\end{array}\right],
$$

where $D_{L(a)}=X_{\mathbb{C}}$.

One thing is to know more detailed information on the eigenvalues of the linearized operator $L(a)$ defined in (6). Notice that the eigenvalues of $-\Delta$ subject to the Neumann boundary condition in $(0, \xi \pi)$ are given by

$$
\rho_{k}=\frac{k^{2}}{\xi^{2}}
$$

with the corresponded eigenfunctions $\phi_{k}(x)=\cos (k x / \xi)$, with $k=0,1,2, \ldots$.

We consider the characteristic problem of $L(a)$ taking in the following form:

$$
L(a)\left(\begin{array}{l}
\phi \\
\varphi
\end{array}\right)=\beta(a)\left(\begin{array}{l}
\phi \\
\varphi
\end{array}\right) .
$$

Setting

$$
\left(\begin{array}{l}
\phi \\
\varphi
\end{array}\right)=\left(\begin{array}{c}
\sum_{k=0}^{\infty} m_{k} \phi_{k}(x) \\
\sum_{k=0}^{\infty} n_{k} \phi_{k}(x)
\end{array}\right)
$$

and introducing it into (8), one has

$$
L_{k}(a)\left(\begin{array}{c}
m_{k} \\
n_{k}
\end{array}\right)=\beta(a)\left(\begin{array}{c}
m_{k} \\
n_{k}
\end{array}\right),
$$

where

$$
L_{k}(a):=\left[\begin{array}{cc}
-\frac{d_{u} k^{2}}{\xi^{2}}+\frac{s a}{b-a}, & s(b-a) \\
-\frac{s b}{b-a}, & -\frac{d_{v} k^{2}}{\xi^{2}}-s(b-a)
\end{array}\right] .
$$

This suggests that the eigenvalues of $L(a)$ can be given by the eigenvalues of the matrix $L_{k}(a), k=0,1,2, \ldots$. For more details on how to derive $L_{k}(a)$, we suggest the interested readers to refer to $[9,12]$ and more references therein for great details.

The characteristic equation of $L_{k}(a)$ for certain fixed $k$ is given by

$$
\rho^{2}-\rho T_{k}(a)+D_{k}(a)=0, \quad(k=0,1,2, \ldots),
$$


where

$$
\begin{aligned}
& T_{k}(\alpha)=\frac{s\left[-a^{2}+(1+2 b) a-b^{2}\right]}{b-a}-\frac{\left(d_{u}+d_{v}\right) k^{2}}{\xi^{2}}, \\
& D_{k}(\alpha)=\frac{d_{u} d_{v} k^{4}}{\xi^{4}}+\frac{s k^{2}}{\xi^{2}}\left[d_{u}(b-a)-\frac{d_{v} a}{b-a}\right]+s^{2}(b-a),
\end{aligned}
$$

where we adopt the same notations of $T_{k}(a)$ and $D_{k}(a)$, which stand for the trace and determinant of $L_{k}(a)$, respectively, as done in [12].

Before proceeding, we state the following useful lemma on the existence of local Hopf bifurcations of the system (3).

Lemma 1 (see [12]). Suppose that, at a certain $a_{0} \in R$, the following condition holds.

$(\mathbb{U})$ There exists a neighborhood $\mathcal{O}$ of $a_{0}$, such that, for any $a \in \mathcal{O}, L(a)$ has a pair of simple, conjugate, and complex eigenvalues $\rho(a)=\alpha(a)+\omega(a)$, continuously differentiable in $a$, with $\alpha\left(a_{0}\right)=0, \omega\left(a_{0}\right)>0$, and $\alpha^{\prime}\left(a_{0}\right) \neq 0$; all the other eigenvalues of $L(a)$ have nonzero real parts.

Then, system (3) has a family of real-valued $T(s)$-periodic solutions $(a(s), u(s)(x, t), v(s)(x, t)), s$ sufficiently small, bifurcating from $\left(a_{0}, a_{0}, b_{0}\right)$. More precisely, there exists a unique $k \in\{0,1,2, \ldots\}$, such that the bifurcating periodic solutions $(a(s), u(s)(x, t), v(s)(x, t))$ can be expressed by

$$
\begin{aligned}
& u(s)(x, t)=s\left[h_{k} e^{2 \pi i t / T(s)}+\overline{h_{k}} e^{-2 \pi i t / T(s)}\right] \cos \left(\frac{k x}{\xi}\right)+o\left(s^{2}\right), \\
& v(s)(x, t)=s\left[g_{k} e^{2 \pi i t / T(s)}+\overline{g_{k}} e^{-2 \pi i t / T(s)}\right] \cos \left(\frac{k x}{\xi}\right)+o\left(s^{2}\right),
\end{aligned}
$$

where

$$
L_{k}\left(a_{0}\right)\left(\begin{array}{c}
m_{k} \\
n_{k}
\end{array}\right)=i \omega\left(a_{0}\right)\left(\begin{array}{c}
m_{k} \\
n_{k}
\end{array}\right) .
$$

In the following, we will use Lemma 1 to prove the existence of the Hopf bifurcations and its corresponding periodic solutions, spatially homogeneous and nonhomogeneous. Clearly, the condition $(\mathbb{W})$ stated in Lemma 1 holds at a certain critical point $a^{H}$ if and only if one of the following conditions holds:

$$
\begin{aligned}
T_{k}\left(a^{H}\right)=0, & D_{k}\left(a^{H}\right)>0 ; \\
T_{j}\left(a^{H}\right) \neq 0, & D_{j}\left(a^{H}\right) \neq 0 \\
\text { for } j \neq k ; & T_{k}^{\prime}\left(a^{H}\right) \neq 0
\end{aligned}
$$

or

$$
\begin{array}{rr}
T_{k}\left(a^{H}\right)=0, & D_{k}\left(a^{H}\right)>0 ; \\
T_{j}\left(a^{H}\right)=0, & D_{j}\left(a^{H}\right)<0 \\
\text { for } j \neq k ; & T_{k}^{\prime}\left(a^{H}\right) \neq 0 .
\end{array}
$$

We define $A(a)=\left(-a^{2}+(1+2 b) a-b^{2}\right) /(b-a)$. Then it is direct to check that, for all $a \in\left(0, a_{0}\right)$, we have $A(a)<0$; and, for all $a \in\left(a_{0}, b\right)$, we have $A(a)>0$, where

$$
a_{0}:=\frac{2 b+1-\sqrt{4 b+1}}{2} .
$$

This implies that, for all $a \in\left(0, a_{0}\right), T_{k}(a)<0$ for all $k \in \mathbb{N}$. Thus, Hopf bifurcation is impossible.

Then, any potential Hopf bifurcation point $\alpha^{H}$ must be in the interval $\left[a_{0}, b\right)$. For any Hopf bifurcation point $a^{H}$ in $\left[a_{0}, b\right)$, let $\alpha(a) \pm i \omega(a)$ be the eigenvalues of $L_{k}(a)$ near $a=a^{H}$; then we have

$$
\begin{gathered}
\alpha(a)=\frac{s\left[-a^{2}+(1+2 b) a-b^{2}\right]}{2(b-a)}-\frac{\left(d_{u}+d_{v}\right) n^{2}}{2 \xi^{2}}, \\
\omega(a)=\sqrt{D_{k}(a)-\alpha^{2}(a),} \\
\alpha^{\prime}\left(a^{H}\right)=\left.\frac{s(b-a)^{2}+s b}{2(b-a)^{2}}\right|_{a=a^{H}}>0 .
\end{gathered}
$$

This implies that $\alpha^{\prime}\left(a^{H}\right) \neq 0$ is always satisfied for any $a^{H} \epsilon$ $\left[a_{0}, b\right)$.

In the following we fix $d_{u}, d_{v}, b, s>0$ but choose $\xi$ appropriately. We notice that

$$
\lim _{a \rightarrow b^{-}} A(a)=\lim _{a \rightarrow b^{-}} \frac{-a^{2}+(1+2 b) a-b^{2}}{b-a}=+\infty .
$$

Then, for any $0 \leq j, A(a)=\left(d_{u}+d_{v}\right) j^{2} / s \xi^{2}$ has positive roots in $\left[a_{0}, b\right)$. We define $\alpha_{j}^{H}$ to be the roots of $A(a)=\left(d_{u}+\right.$ $\left.d_{v}\right) j^{2} / s \xi^{2}$ satisfying $0<a_{0}^{H}<a_{j}^{H}<\beta$ and $\lim _{j \rightarrow \infty} a_{j}^{H}=b$. These points satisfy

$$
0<a_{0}=a_{0}^{H}<a_{1}^{H}<a_{2}^{H}<\cdots<a_{n}^{H}<\cdots<b .
$$

Clearly $T_{j}\left(a_{j}^{H}\right)=0$ and $T_{i}\left(a_{j}^{H}\right) \neq 0$ for $i \neq j$. Now we only need to verify whether $D_{i}\left(a_{j}^{H}\right) \neq 0$ for all $i \in\{0,1,2, \ldots\}$ and, in particular, $D_{j}\left(a_{j}^{H}\right)>0$.

In fact, if the discriminant of $D_{i}(a)$ is less than 0 , then we can get $D_{i}(a)>0$ for all $i \in\{0,1,2, \ldots\}$ and, in particular, $D_{j}\left(a_{j}^{H}\right)>0$. The discriminant of $D_{i}(a)$ is given by

$$
\Delta=s^{2}\left\{\left[d_{u}(b-a)-\frac{d_{v} a}{b-a}\right]^{2}-4 d_{u} d_{v}(b-a)\right\},
$$

which is negative when $\alpha \in\left[a_{0}^{H}, a_{c}\right)$, where

$$
a_{c}:=\frac{2 d_{u} b-d_{v}-2 \sqrt{d_{u} d_{v} b}+\sqrt{d_{v}^{2}+4 d_{2} \sqrt{d_{u} d_{v} b}}}{2 d_{u}} .
$$

Summarizing our analysis above, we obtain our main result in this subsection. 
Theorem 2. Let $a_{j}^{H}, j \in\{0,1,2, \ldots\}$ be defined above. Then, at any $a_{j}^{H}$ satisfying $a_{j}^{H} \in\left[a_{0}^{H}, a_{c}\right)$, the system (1) undergoes a Hopf bifurcation at $\alpha=\alpha_{j}^{H}$. Moreover,

(1) the bifurcating periodic solutions from $a=a_{0}^{H}$ are spatially homogeneous, which coincides with the periodic solution of the corresponding ODE system;

(2) the bifurcating periodic solutions from $a=a_{j}^{H}, j \in$ $\{1,2, \ldots\}$, are spatially nonhomogeneous.

Proof. By the analysis above, we know that the condition (17) holds true (while (16) does not). This implies that the condition $(\mathbb{U})$ in Lemma 1 is satisfied. Thus, by Lemma 1 , the local Hopf bifurcation occurs near $\left(a_{j}^{H}, u_{a_{j}^{H}}, v_{a_{j}^{H}}\right)$ at $a=a_{j}^{H}$, with $j \in\{0,1,2, \ldots\}$. By the expression of (14), if $k=0$, then the solution depends only on the time variable $t$; thus, the periodic solution is exactly the same as that of the periodic solutions in the corresponding ODEs system. However, if $k \geq 1$, then the periodic solutions depend not only on $x$ but also on $t$ and thus are spatially nonhomogeneous.

\section{Stability of the Spatially Homogeneous Periodic Solutions Bifurcating from $a=a_{0}^{H}$}

In this section, we consider the Hopf bifurcation direction and the stability of the bifurcating spatially homogeneous periodic solutions from the Hopf bifurcation points $a_{0}^{H}$.

Theorem 3. For the system (1), the Hopf bifurcation at $a=$ $a_{0}^{H}$ is subcritical, and the bifurcating (spatial homogeneous) periodic solutions are unstable.

Proof. By Theorem 2.1 of [12], in order to determine the stability and bifurcation direction of the bifurcating periodic solution, we need to calculate $\operatorname{Re}\left(c_{1}\left(a_{0}^{H}\right)\right)$. When $a=a_{0}^{H}$, we put

$$
\begin{gathered}
q:=\left(\begin{array}{c}
m_{0} \\
n_{0}
\end{array}\right)=\left(\begin{array}{c}
1 \\
-1+\frac{i \omega_{0}}{s\left(b-a_{0}^{H}\right)}
\end{array}\right), \\
q^{*}:=\left(\begin{array}{c}
m_{0}^{*} \\
n_{0}^{*}
\end{array}\right)=\left(\begin{array}{c}
\frac{1}{2 \xi \pi}+\frac{i s\left(b-a_{0}^{H}\right)}{2 \omega_{0} \xi \pi} \\
\frac{i s\left(b-a_{0}^{H}\right)}{2 \omega_{0} \xi \pi}
\end{array}\right),
\end{gathered}
$$

where $\omega_{0}=s \sqrt{b-a_{0}^{H}}$.

As in Section 2 of [12], we define

$$
\begin{gathered}
f(a, u, v)=s\left[\left(u+u_{a}\right)\left(v+v_{a}\right)-\left(u+u_{a}\right)-a\right], \\
g(a, u, v)=s\left[b-\left(u+u_{a}\right)\left(v+v_{a}\right)\right]
\end{gathered}
$$

then we have, by (2.19) of [12], (we use the same notations of $c_{0}, d_{0}, e_{0}, f_{0}, g_{0}$ as those appeared in [12])

$$
\begin{aligned}
& c_{0}=-d_{0}=2 s\left[-1+\frac{i \omega_{0}}{s\left(b-a_{0}^{H}\right)}\right], \\
& e_{0}=-f_{0}=-2 s, \quad g_{0}=h_{0}=0 .
\end{aligned}
$$

Thus, as in [12], we need to calculate $\left\langle q^{*}, Q_{q q}\right\rangle,\left\langle q^{*}, Q_{q \bar{q}}\right\rangle$, $\left\langle\bar{q}^{*}, Q_{q q}\right\rangle$, and $\left\langle q^{*}, C_{q q \bar{q}}\right\rangle$. They are

$$
\begin{gathered}
\left\langle q^{*}, Q_{q q}\right\rangle=\int_{0}^{\xi \pi} \bar{q}^{* T}\left(c_{0}, d_{0}\right)^{T} d x=\frac{i \omega_{0}}{b-a_{0}^{H}}-s, \\
\left\langle q^{*}, Q_{q \bar{q}}\right\rangle=\int_{0}^{\xi \pi} \bar{q}^{* T}\left(e_{0}, f_{0}\right)^{T} d x=-s, \\
\left\langle\bar{q}^{*}, Q_{q q}\right\rangle=\int_{0}^{\xi \pi} \bar{q}^{* T}\left(c_{0}, d_{0}\right)^{T} d x=\frac{i \omega_{0}}{b-a_{0}^{H}}-s, \\
\left\langle q^{*}, C_{q q \bar{q}}\right\rangle=\int_{0}^{\xi \pi} \bar{q}^{* T}\left(g_{0}, h_{0}\right)^{T} d x=0 .
\end{gathered}
$$

Hence it is straightforward to calculate

$$
\begin{aligned}
& H_{20}=\left(\begin{array}{c}
c_{0} \\
d_{0}
\end{array}\right)-\left\langle q^{*}, Q_{q q}\right\rangle\left(\begin{array}{c}
m_{0} \\
n_{0}
\end{array}\right)-\left\langle\bar{q}^{*}, Q_{q q}\right\rangle\left(\begin{array}{c}
\overline{m_{0}} \\
\overline{n_{0}}
\end{array}\right)=0, \\
& H_{11}=\left(\begin{array}{c}
e_{0} \\
f_{0}
\end{array}\right)-\left\langle q^{*}, Q_{q \bar{q}}\right\rangle\left(\begin{array}{c}
m_{0} \\
n_{0}
\end{array}\right)-\left\langle\bar{q}^{*}, Q_{q \bar{q}}\right\rangle\left(\begin{array}{c}
\overline{m_{0}} \\
\overline{n_{0}}
\end{array}\right)=0 \text {, }
\end{aligned}
$$

which implies that $w_{20}=w_{11}=0$. So,

$$
\left\langle q^{*}, Q_{w_{11} q}\right\rangle=\left\langle q^{*}, Q_{w_{20} \bar{q}}\right\rangle=0 .
$$

Therefore, after the steps of calculations, we obtain

$$
\begin{aligned}
\operatorname{Re}\left(c_{1}\left(a_{0}^{H}\right)\right) & =\operatorname{Re}\left\{\frac{i}{2 \omega_{0}}\left\langle q^{*}, Q_{q q}\right\rangle \cdot\left\langle q^{*}, Q_{q \bar{q}}\right\rangle+\frac{1}{2}\left\langle q^{*}, C_{q q \bar{q}}\right\rangle\right\} \\
& =\frac{s}{2\left(b-a_{0}^{H}\right)}>0 .
\end{aligned}
$$

From (20), it follows that $\alpha^{\prime}\left(a_{0}^{H}\right)>0$, and then by Theorem 2.2 of [12] the bifurcation is subcritical. On the other hand, from (13), $T_{k}\left(a_{0}^{H}\right)<0$ and $D_{k}\left(a_{0}^{H}\right)>0$ for any $n \geq 1$, so the bifurcating periodic solutions are unstable since $\operatorname{Re}\left(c_{1}\left(a_{0}^{H}\right)\right)>0$.

\section{Conclusions}

In this paper, by using the standard Hopf bifurcation theorem, we are able to prove the existence of periodic solutions of the system. And we also proved that the spatial homogeneous periodic solution bifurcating from the smallest Hopf bifurcation point $a_{0}^{H}$ is unstable. By [12], the spatially nonhomogeneous periodic solutions bifurcating from $a_{j}^{H}, j \in$ $\{1,2, \ldots\}$, are all unstable. This together with our results shows that all the Hopf bifurcating periodic patterns are unstable. 


\section{Conflict of Interests}

The authors declare that there is no conflict of interests regarding the publication of this paper.

\section{Acknowledgment}

The authors are grateful to the anonymous referee for the comments and suggestions which definitely led to an improved presentation of the paper.

\section{References}

[1] A. Turing, "The chemical basis of morphogenesis," Philosophical Transactions of the Royal Society B, vol. 237, pp. 37-72, 1952.

[2] P. de Kepper, V. Castets, E. Dulos, and J. Boissonade, “Turingtype chemical patterns in the chlorite-iodide-malonic acid reaction," Physica D: Nonlinear Phenomena, vol. 49, no. 1-2, pp. 161-169, 1991.

[3] I. R. Epstein and J. A. Pojman, An Introduction to Nonlinear Chemical Dynamics, Oxford University Press, 1998.

[4] I. Lengyel and I. R. Epstein, "Modeling of turing structures in the chlorite-iodide-malonic acid-starch reaction system," Science, vol. 251, no. 4994, pp. 650-652, 1991.

[5] I. Lengyel and I. R. Epstein, "A chemical approach to designing Turing patterns in reaction-diffusion systems," Proceedings of the National Academy of Sciences of the United States of America, vol. 89, no. 9, pp. 3977-3979, 1992.

[6] J. Jang, W.-M. Ni, and M. Tang, "Global bifurcation and structure of Turing patterns in the 1-D Lengyel-Epstein model," Journal of Dynamics and Differential Equations, vol. 16, no. 2, pp. 297-320, 2004.

[7] J. D. Murray, Mathematical Biology. I. An Introduction, Interdisciplinary Applied Mathematics, Springer, New York, NY, USA, 3rd edition, 2002.

[8] J. D. Murray, Mathematical Biology II. Spatial Models and Biomedical Applications, Interdisciplinary Applied Mathematics, Springer, New York, NY, USA, 2003.

[9] W.-M. Ni and M. Tang, "Turing patterns in the Lengyel-Epstein system for the CIMA reaction," Transactions of the American Mathematical Society, vol. 357, no. 10, pp. 3953-3969, 2005.

[10] S. Ruan, "Diffusion-driven instability in the Gierer-Meinhardt model of morphogenesis," Natural Resource Modeling, vol. 11, no. 2, pp. 131-142, 1998.

[11] A. R. Sanderson, R. M. Kirby, C. R. Johnson, and L. Yang, "Advanced reaction-diffusion models for texture synthesis," Journal of Graphics Tools, vol. 11, no. 3, pp. 47-71, 2006.

[12] F. Yi, J. Wei, and J. Shi, "Bifurcation and spatiotemporal patterns in a homogeneous diffusive predator-prey system," Journal of Differential Equations, vol. 246, no. 5, pp. 1944-1977, 2009.

[13] F. Yi, J. Liu, and J. Wei, "Spatiotemporal pattern formation and multiple bifurcations in a diffusive bimolecular model," Nonlinear Analysis: Real World Applications, vol. 11, no. 5, pp. 3770-3781, 2010.

[14] D. Henry, Geometric Theory of Semilinear Parabolic Equations, Lecture Notes in Mathematics, Springer, New York, NY, USA, 1980. 


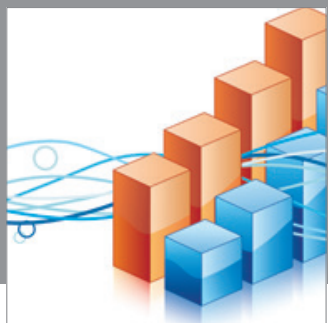

Advances in

Operations Research

mansans

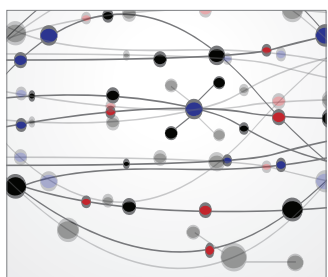

The Scientific World Journal
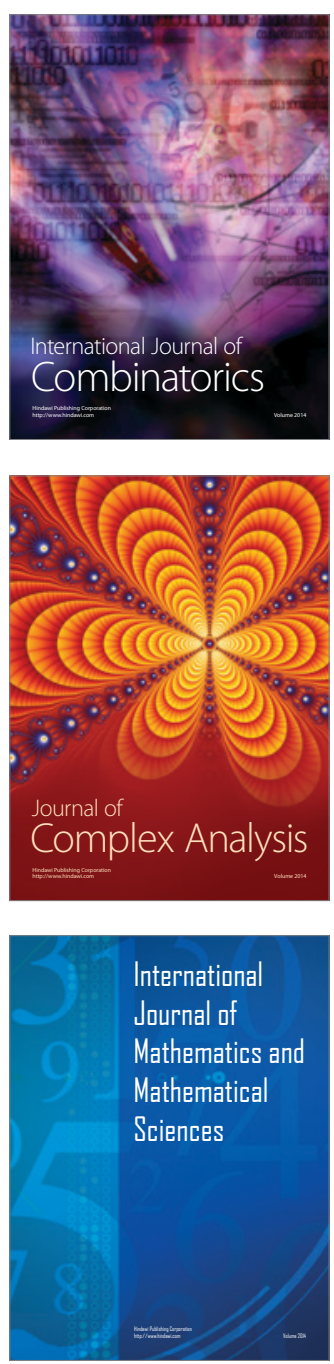
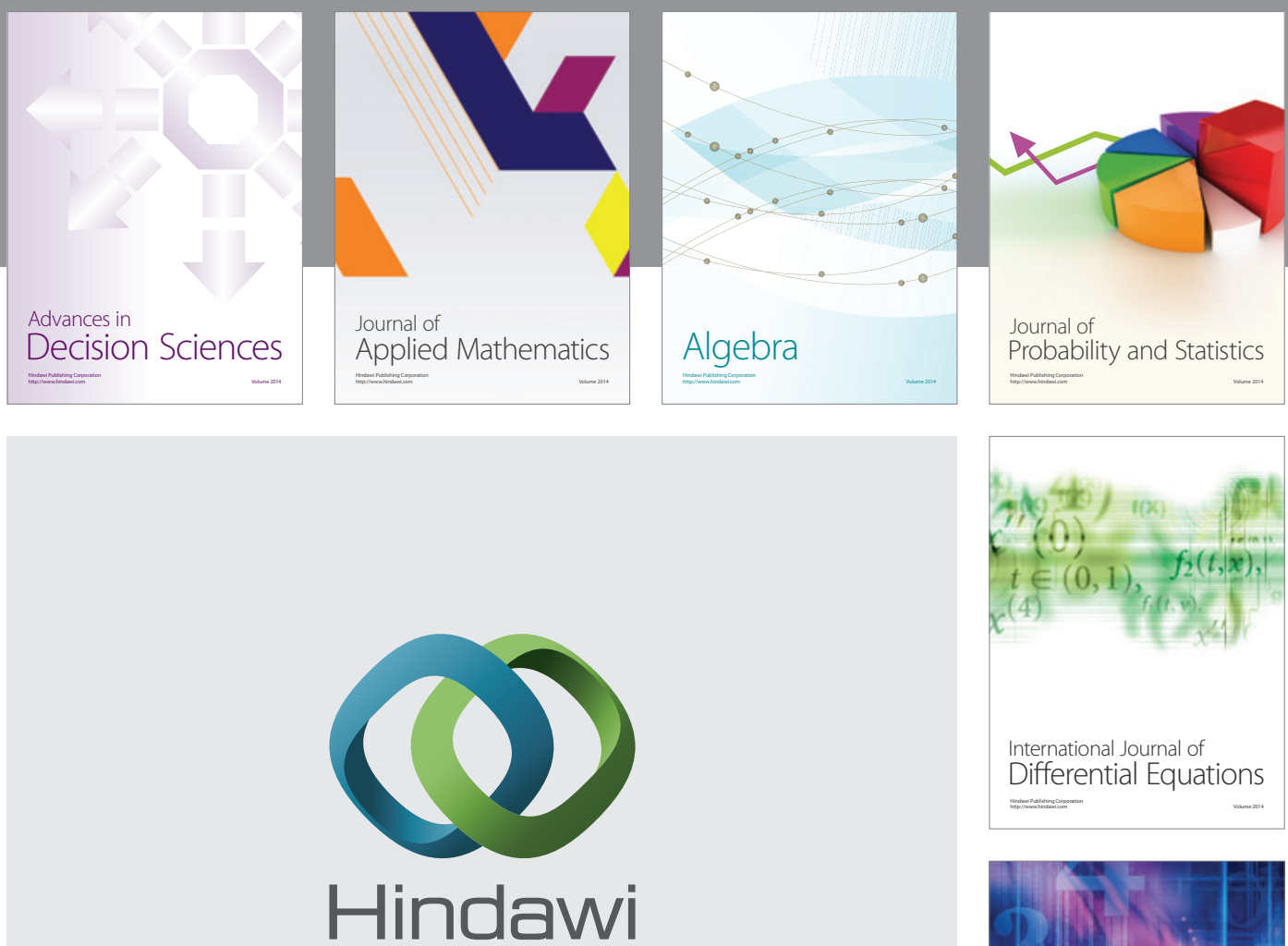

Submit your manuscripts at http://www.hindawi.com
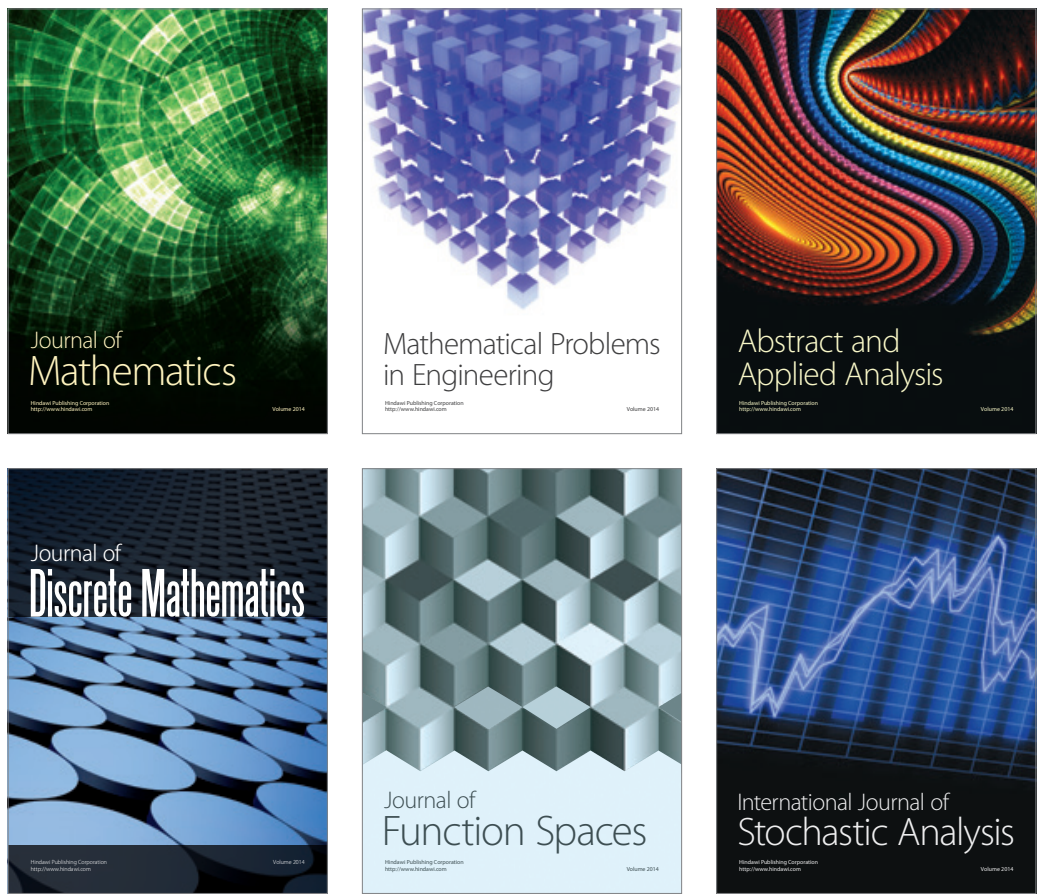

Journal of

Function Spaces

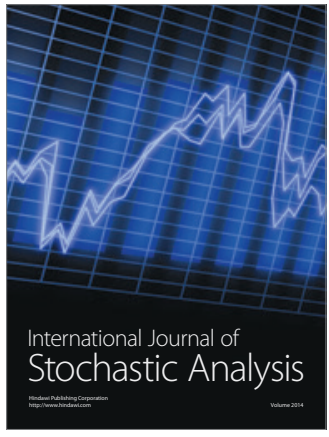

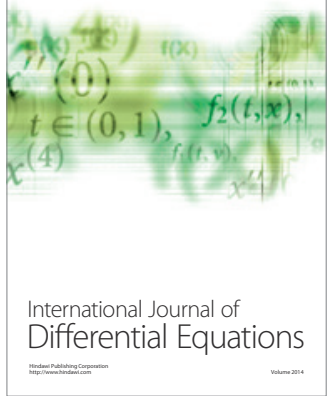
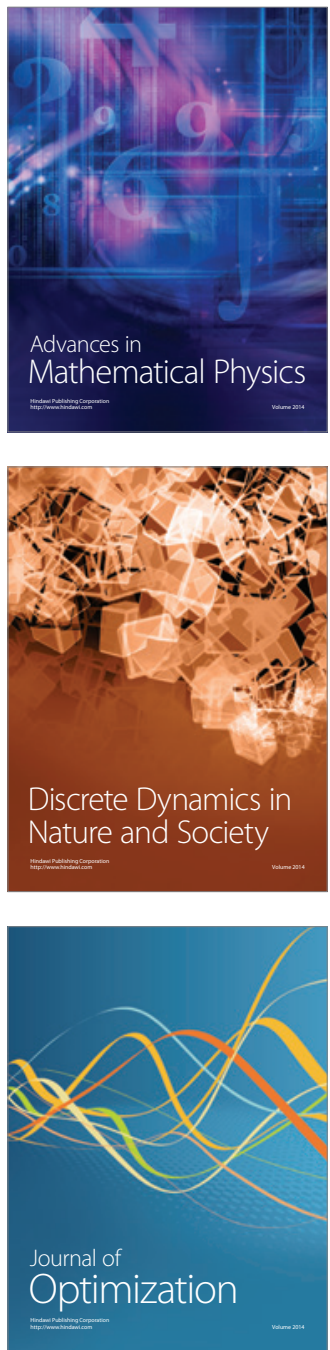